\title{
Erratum
}

\section{Erratum to: A study of volatile organic compound concentrations in a new school building over one year period}

For the article "A study of volatile organic compound concentrations in a new school building over one year period" published in 4(4): 248-255, 2012, DOI: 10.1007/s13530-012-0143-4, the authors would like to remove an author name (Dr. Chung-Sik Yoon) from the author list. The original author list in this article was incorrect which was caused by an inadvertent editing of the corresponding author. The corrected authorship should be "Lim-Kyu Lee, Ju-Hyun Kim, Bokyung Kim, and Junghwan Kim”. The authors sincerely apologize and regret any inconvenience caused. 